\title{
COMMENTARY
}

\section{Tailoring the process of informed consent in genetic and genomic research}

\author{
Charles N Rotimi*1 and Patricia A Marshall*2
}

\begin{abstract}
Genomic science and associated technologies are facilitating an unprecedented rate of discovery of novel insights into the relationship between human genetic variation and health. The willingness of large numbers of individuals from different ethnic and cultural backgrounds to donate biological samples is one of the major factors behind the success of the ongoing genomic revolution. Although current informed consent documents and processes demonstrate a commitment to ensuring that study participants are well informed of the risks and benefits of participating in genomic studies, there continues to be a need to develop effective new approaches for adequately informing participants of the changing complexities of the scientific and ethical issues that arise in the conduct of genomics research. Examples of these complexities in genomic research include more widespread use of wholegenome sequencing technologies, broad sharing of individual-level data, evolving information technology, the growing demand for the return of genetic results to participants, and changing attitudes about privacy and the expansion of genomics studies to global populations representing diverse cultural, linguistic and socio-economic backgrounds. We highlight and briefly discuss the importance of ten core scientific, cultural and social factors that are particularly relevant to tailoring informed consent in genomic research, and we draw attention to the need for the informed consent document and process to be responsive to the evolving nature of genomic research.
\end{abstract}

*Correspondence: rotimic@mail.nih.gov, patricia.marshall@case.edu 'Inherited Disease Research Branch, Center for Research on Genomics and Global Health, National Human Genome Research Institute, National Institutes of Health 12 South Drive, Bethesda, MD 20892-5635, USA. ${ }^{2}$ Department of Bioethics, School of Medicine, Case Western Reserve University, 11009 Euclid Avenue, Cleveland, $\mathrm{OH} 44106$, USA

\section{Introduction}

Advances in genomic technology and computational approaches have significantly changed our understanding of the non-random distribution of human genetic variants and its impact on disease susceptibility and variable drug response across human populations. A critical element of this success story has been the availability of large cohorts of unrelated individuals and families willing to donate tissue and blood samples for genetic and biochemical analysis. Increasingly, genomic studies are being conducted among people from diverse cultural, linguistic and socio-economic backgrounds throughout the world. The global expansion of genomic research, combined with the rapid evolution of scientific knowledge and the public health need to translate genomic findings, show the importance of continued development of new, effective approaches to the process of informed consent. Here, we use national and international projects to illustrate the growing complexities of scientific and ethical issues in genomics and their implications for informed consent. Tailored approaches to the informed consent process need to address both the scientific and regulatory constraints of designing and implementing genomic research, and the experiences, knowledge and concerns of individuals and diverse communities invited to join genetic research projects.

\section{Points to consider in tailoring informed consent to genetic research}

National and international policies and guidelines address a broad range of issues regarding ethical conduct in genetic and genomic studies [1-6]. These policies and recommendations, and legislation such as the US Genetic Nondiscrimination Information Act (GINA) of 2008 [7], focus attention on topics ranging from the collection and storage of samples [8], data sharing for research purposes [9-11], protection of individual privacy [12-14], and the process and documentation of informed consent [15-18]. Our objective here is to highlight and briefly describe the importance of ten core scientific, cultural and social factors that are particularly relevant to designing ethically responsible approaches to informed consent in genomic research involving ethnically, socio-economically and linguistically diverse study populations globally (Table 1). 


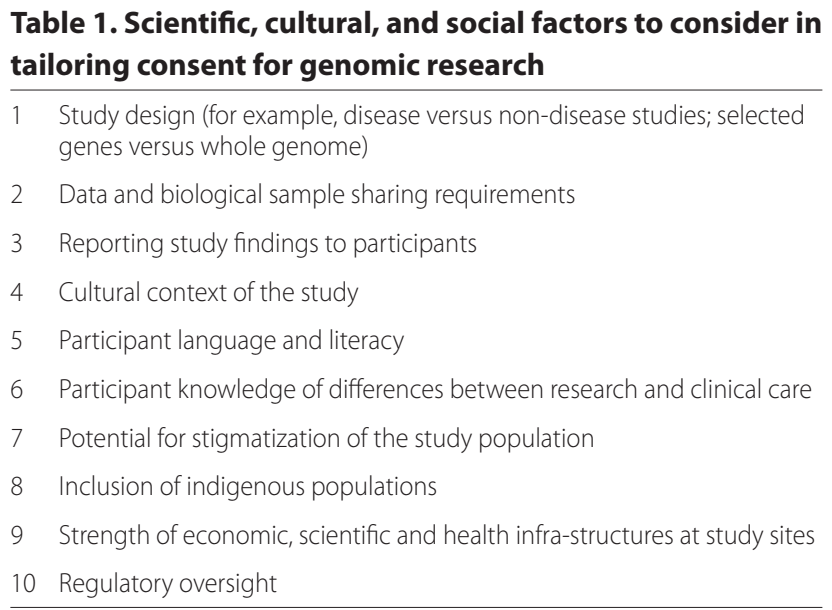

\section{Study design}

Protocols for genomic research differ considerably depending on the study aims, sample populations and the procedures, risks and benefits associated with the research. The particular study design and the relationship of investigators to individuals and communities involved in the project have implications for the obligations of researchers to study participants; this in turn influences the substance and process of informed consent. For example, the International HapMap Project [19] involved the collection of anonymized samples to identify and catalog genetic similarities and differences in human beings. Providing personal feedback to participants about genetic results in the HapMap project was therefore not a possibility. In contrast, large-scale medical genotyping and sequencing research studies such as the pioneering National Institutes of Health (NIH) medical sequencing project called ClinSeq [20,21], which is designed to investigate how to do genome sequencing in clinical research, will provide genetic and clinical information to participants. To accommodate this study design, the informed consent process for ClinSeq addresses complex issues regarding procedures for communicating information and the implications for individuals who receive the results.

The informed consent process may need to emphasize additional or different factors in other types of genetic research. For example, obligations of the investigator to the participants differ in case-control genomic studies involving unrelated individuals compared with family studies; issues surrounding paternity, for example, are not directly relevant in genomic studies of unrelated individuals. Other questions that influence approaches to the process and content of informed consent arise in studies exploring genetic information obtained from specific genetic variants (such as single nucleotide polymorphisms) within one or a few genes compared with the whole genome. The creation of cell lines presents yet another different set of issues, including the availability of an unlimited supply of genetic materials for an undefined period of time.

Overall, the design of genomic studies is perhaps the single most important factor that shapes the informed consent document and process. Beliefs and concerns associated with different types of genomic research vary among diverse population groups throughout the world. Therefore, investigators should consider carefully the underlying local social and cultural issues that are relevant to the design of genetic research when preparing documents and approaches to the consent process.

\section{Data and biological sample sharing}

The ability to combine and share large datasets generated by genomic projects has contributed significantly to the success stories enjoyed by the genomic scientific communities. This is so because genomic techniques such as the agnostic search of the genomes of individuals with disease compared with those without disease (called a genome-wide association study, GWAS) requires large numbers of study participants, usually in the thousands, to have adequate statistical power to find an association if one exists. These large datasets containing demographic, clinical and genetic information are usually deposited in data repositories such as dbGaP [22] with two main types of access requirements - fully open or controlled-access. The fully open databases (such as the International HapMap Project and the 1000 Genomes Project [19] generated from non-identifiable samples) can be directly accessed and downloaded via the internet by anyone, without any restriction [22]. Fully open databases are anonymized and do not contain clinical (phenotype) information except gender and ethnicity/ancestry. In contrast, controlled-access databases such as GWASs may contain individual-level demographic, clinical and genetic information; to access these controlled databases, investigators are required to obtain permission from a data access committee. Although these types of database are coded and de-identified and therefore do not contain information that is traditionally used to identify individuals (such as name, address, and telephone and social security number), there is a possibility that someone may develop ways to link information contained within them to individual research subjects. Because of this possibility and government policies such as the NIH GWAS Policy [23] that require study subjects to be informed that their phenotype and genotype data will be shared for research purposes, the informed consent documents for these studies are expected to be tailored to contain appropriate language to enable study participants to make informed decisions regarding broad data sharing. Complications associated with the ability to withdraw from studies will become 
increasingly problematic, especially after broad release of data, and this issue will need to be carefully assessed in approaches to the consent process $[17,18]$.

International collaborative genomic studies involving data and sample sharing between high- and low-income countries call attention to additional ethical and social justice issues. For example, communicating information about the complex implications of sharing genetic and phenotypic information that may have implications for participants and their families must be addressed using language in the consent process that is both culturally meaningful and comprehensive. Moreover, as investigators involved in the MalariaGen project point out [24], it is important to ensure that scientists in developing countries are not compromised because of the timing of the public release of data to the global scientific community. In this situation, open access to the data could place researchers from developing countries at a disadvantage because they might not have the resources or capacity to respond as quickly to the data as scientists in developed countries. For this reason, MalariaGen investigators have instituted a policy that includes capacity building and training for scientists in low-income settings involved in their genomic research [24].

Traditionally, consent for genetic and genomic research has addressed the issue of sample sharing by asking participants to choose whether they want to limit the sample use to only the current study or disease under investigation, or be re-contacted for future studies, or if they would allow future use of samples without recontact. However, these options have their limitations and raise several questions. For example, it may be difficult for study participants to make judgments about future use because it is hard to fully comprehend the implications of such decisions given the rapidly changing landscape of biomedical research in general, and genomic science in particular.

\section{Reporting study results to participants}

In the past, most genomic research projects did not report results back to participants. This decision was due, for the most part, to the uncertain clinical relevance of research findings. It is, however, becoming increasingly difficult to justify this position, especially in the context of large-scale medical genotyping and sequencing research studies that are likely to generate clinically relevant genetic information. Examples of this type of genomic study include ClinSeq [20,21], the Coriell Personalized Medicine Collaborative [25], the Framingham Heart Study [26] and the Jackson Heart Study [27]. However, communicating genomic results to participants requires tailored consent documents that carefully consider ethical responsibilities and social obligations to participants and their relatives. To address these issues, the consent process and documents must contain clear and appropriate language that communicates the risks and benefits of receiving genetic information likely to have varying levels of clinical and socio-economic relevance to study subjects, their relatives and ethnic groups. Also, the ability to successfully use the genetic information to inform individual and public health will depend on many cultural and socio-economic factors. For example, low levels of literacy and access to care especially the availability of genetic counselors in a resource-poor environment - pose significant challenges to investigators who may have good intentions about reporting results or are required by law to communicate genetic results to study participants.

An important consideration in genomic projects such as ClinSeq $[20,21]$ is the discovery of clinically actionable results that are not part of the original aim of the study. For example, because ClinSeq is conducting complete sequencing of hundreds of cardiovascular genes, investigators may discover genetic variants that have implications for non-cardiovascular diseases, such as cancer. What are ClinSeq investigators' ethical and legal obligations to communicate incidental results to participants? How should this information be communicated to participants? Although study participants may want to obtain results, what can or should they do with the information [28]? Social and political conundrums surrounding differential access to health care and health inequalities between population groups exacerbate challenges associated with disclosing both intended and unanticipated genetic findings. These and similar issues must be anticipated and adequately addressed in the informed consent process and documents.

The ClinSeq consent document [20,21] is a good example of tailoring the informed consent process to explain issues related not only to communicating results ranging from genetic variants known to cause disease to novel and uncertain genetic variants with no known biological meaning - but also the potential psychological problems if participants learn they are carriers of clinically relevant genetic variants that have implications for themselves and family members. For example, the ClinSeq consent document [21] contains specific language about the availability of genetic counselors to participants who may experience psychological problems as a result of knowing that they carry genetic variants that may increase their risk of disease. Current debates over whether or not to report these findings, and questions raised about procedures for reporting, reflect the complexity of the underlying concerns [28-32].

\section{Cultural context}

Beliefs associated with illness experiences, inherited diseases and biomedical and genetic research are 
embedded in cultural values and traditions that may have implications for the implementation of genomic studies and the design of consent processes [33]. Participants may have personal, religious or ethical beliefs that limit the types of medical tests, treatments or procedures they would want to receive as part of study participation (vaccination and blood transfusion, for example). In some cultural settings, customs and traditions also influence beliefs about who has the authority to provide informed consent for research participation [34-37]. For example, in our genomic research on podoconiosis in Southern Ethiopia [38], we found that participants wanted to discuss the study with family members before giving consent. Similarly, in our international project investigating factors influencing informed consent for genetic research on hypertension in a rural town in Nigeria [39], we found that nearly half of the married women reported that they needed to talk with their husbands before giving their consent.

\section{Language and literacy}

The language spoken by study participants and literacy levels of study populations are essential factors to consider in developing tailored approaches to informed consent. Although it may seem obvious for investigators to develop linguistically appropriate consent documents using clear and simple language, the use of complicated biomedical and scientific language, and lengthy and cumbersome consent forms, continue to be challenging for participants, particularly in low-income settings around the world [40,41]. Comprehension of information provided in consent forms and consent discussions is foundational to voluntary participation. How much information is necessary - and in what format - for individuals to understand the implications of joining a genomic study? These are important issues to consider in tailoring informed consent processes for genetic and genomic research. For example, in our podoconiosis project [38], we observed that the majority of participants did not understand that information in the informed consent document and discussion was provided to enable them to make a decision about participating in the study. Instead, participants thought the information was provided as a form of health education.

\section{Participant knowledge of differences between research and clinical care}

A thorny problem for all scientific and medical researchers, not just those involved in genomic studies, concerns misunderstandings about the difference between medical testing or treatment and medical research. Research projects often include procedures to classify the health (disease) status of study participants. These procedures could range from basic clinic activities (such as completing questionnaires, measuring blood pressure and drawing blood) to more involved procedures, such as echocardiograms and computer tomography. There is potential for therapeutic misconception, and this is a serious challenge for investigators. The important issue here is that, in some studies, diagnostic services could represent clinical services for participants; this may be both an incentive and a source of confusion for individuals, particularly in settings in which medical care is limited or unavailable. The Framingham Heart Study [26], the Jackson Heart Study [27], the Coriell Personalized Medicine Collaborative Study [25] and ClinSeq $[20,21]$ are all examples of projects in which participants derive direct benefits because they will undergo testing that could lead to clinically relevant information such as disease diagnosis. In contrast, studies like the International HapMap Project [19] and our genetics of podoconiosis study in Ethiopia [38] do not provide direct clinical benefits to participants. Regardless of direct or indirect study benefits, it is important to develop linguistically and culturally meaningful approaches to informed consent to ensure that participants know they are involved in a genetic research project and not undergoing tests or procedures for clinical care.

\section{Potential for stigmatization of study populations}

A tailored informed consent process should consider the social meaning that study participants attach to the disease under investigation. Diseases such as hypertension or diabetes may be viewed very differently from potentially stigmatizing conditions such as mental illnesses or physically identifiable diseases. Podoconiosis, for example, is a disease that results in the swelling of the lower legs among people exposed to red clay soil. It is a stigmatizing health condition in endemic areas such as Ethiopia because of the widely held beliefs that the disease runs in families and is untreatable. We recently demonstrated [42] that the social stigma attached to podoconiosis affected the process of obtaining informed consent for genetic research on this disease in Southern Ethiopia; we found that participants were afraid of participating in a genetic study because they were fearful that it might aggravate stigmatization by exposing the familial nature of the disease. Investigators have a responsibility to identify additional risks associated with genetic research participation for stigmatized individuals or groups when developing approaches for informed consent, and should also use culturally appropriate strategies to protect marginalized groups [43,44]. Before initiating a study, researchers should consider what confidentiality, privacy and 'secrecy' mean to study participants who may bear the burden of stigmatization or discrimination, and they should apply this knowledge in developing the consent process. 


\section{Indigenous populations}

Genetic investigators working with indigenous populations face unique challenges. For example, some researchers and industries have been accused of 'biopiracy' by engaging in research activities that disrespect or take unfair advantage of ownership of indigenous biological resources. Biopiracy often leads to inadequate compensation to the people - or nations - who provided the biological samples. Accusations of biopiracy, whether or not the allegations are true, can affect both the willingness of indigenous groups to participate in research and the enthusiasm of scientists to approach indigenous communities about participating in genomic research. It is therefore important that issues surrounding biopiracy are addressed before the initiation of sample and data collection. It is also essential that intellectual property rights and the development of patents are addressed before initiating genomic research with indigenous groups [45]. Benefits derived from genetic research include financial gain associated with product development and patents based on study results, and this has direct implications for future obligations of investigators at the completion of a project [46]. For example, in 2000, AutoGen, an Australian biotechnology firm, signed an agreement with the Ministry of Health in Tonga to establish a private genetic database to study genes involved in diabetes, obesity and other diseases [47]. Although ownership of the DNA samples would be the property of Tonga, AutoGen would retain exclusive rights to the database and could use it for research that would lead to drug development. In return, AutoGen would provide Tonga's Ministry of Health with annual research funding and royalties from commercialized products based on gene discoveries; pharmaceutical drugs developed would be provided for free to the Ministry of Health. Serious ethical questions were raised over issues associated with privacy, ownership and the commercialization of genetic material in a resource-poor setting such as Tonga, which is ruled by an island monarchy. In 2002, AutoGen indicated that they would not pursue the development of a genetic database in Tonga [47-49].

Another important issue in the context of working with indigenous groups concerns the need, in some cases, for community approval or 'consent', depending on local governance and political authority [50]. Examples of policies for ethical conduct in research that demonstrate respect for the concerns and rights of indigenous populations include guidance for the First Nation people in Canada [51,52], American Indian Nations in the USA [53], aboriginal communities in Australia [54] and the Maori of New Zealand [55].

\section{Socio-economic and health infrastructure}

The strength of economic, scientific and health infrastructures at study sites highlights the need for genomic investigators to pay careful attention to these issues as part of informed consent requirements. In resource-poor environments and low-income settings, researchers may have considerable power to influence the voluntary participation of individuals and communities that they hope to involve in their studies. For example, physicians and other health professionals conducting a research project may also be responsible for the care of potential participants. Also, in some cases, the opportunities for economic support and capacity building that genetic researchers may be able to provide can influence the willingness of local investigators to sponsor the study. Moreover, the effect of unequal power between researchers from resource-rich settings and host sponsors at resource-poor sites may influence local research ethics committees to approve studies and provide regulatory oversight. Questions surrounding the potential for undue influence and its ability to affect voluntary participation must be taken into account. Collaborative partnerships that endure over time contribute to a foundation of trust, cooperation and capacity building; these partnerships help diminish the potential for undue influence at all levels [56].

\section{Regulatory oversight}

Regulatory governance and oversight for genetic and genomic research have direct implications for the preparation of informed consent documents. For example, the implementation of the HapMap Project in Nigeria required approval from three institutional review boards (IRBs) [19]. Although the informed consent document for the International HapMap Project underwent extensive review and revision at the NIH before initiating the study, two of the IRBs - one in the US and the other at the Nigerian site - raised questions about the consent document and requested revisions. Addressing the bureaucratic exigencies of IRBs resulted in the delay of the project [19]. Another example of the impact of regulatory requirements for informed consent concerns the question of whether or not de-identified samples are considered to be 'human subjects'; guidance on this issue will affect the use of samples in future research [18]. Moreover, in multi-national genetic research projects, national regulatory guidelines concerning the definition of human subjects may be in conflict.

\section{Conclusions}

Social and ethical issues associated with the process of informed consent for genomic research are challenging for research participants, investigators and policy makers. We agree with other investigators $[17,18]$ who recognize that policy and guidelines need to be revised quickly in response to the continually evolving enterprise of genomic research as new knowledge is generated and 
technologies advance. Revisions to existing guidelines or the development of new policies will affect the development of informed consent documents. Moreover, it is reasonable to expect that as researchers continue to improve approaches to consent - including clear descriptions of the risks and benefits - individuals may be more likely to donate DNA samples for genomic research or, minimally, may be better informed to make decisions regarding participation in genomic studies.

There is a great need for continuing efforts to increase public knowledge about genomic research. As individuals and communities from diverse social backgrounds become more aware of genomic research and the potential role of genetics in contributing to health outcomes, the public will hopefully be more informed about the implications of genomic research for personal medical care, public health and more broadly the public representation of diverse population groups based on genetic findings. This knowledge should reinforce the ability of potential participants to make informed choices about joining a genetic study. There are complicated issues underlying public trust in medicine as well as scientific and genetic research that must be addressed. Innovative strategies for public education and community engagement should take into account cultural settings and historical experiences that have contributed to distrust in the past.

Finally, there is a critical need for further empirical research on innovative approaches to the process of informed consent for genomic research that take into account scientific, social and cultural factors. Examples of such studies might include randomized trials testing the effectiveness of tailored models of informed consent for different types of genomic studies with socially and ethnically diverse populations. Research exploring the use of simplified consent documents for genetic research, along with pre-consent educational sessions and the provision of educational materials, are another avenue for research. Studies might also examine the effects of using multiple media - such as video tapes, written documents and group or individual educational sessions - on comprehension of study goals, risks, benefits and future implications of participating in a genetic study.

We are at an important juncture in conducting translational genomic research that has potential for clinical and public health applications. Our challenge is to develop approaches to the informed consent process that enhance understanding of the nature, goals and implications of particular studies and simultaneously address the pragmatic constraints of implementing genomic research and reporting study results.

\section{Abbreviations}

GWAS, genome-wide association study; IRB, institutional review board; $\mathrm{NIH}$, National Institutes of Health.
Competing interests

The authors declare that they have no competing interests.

\section{Authors' contributions}

CNR and PAM contributed equally to the design, conceptualization and preparation of the manuscript.

\section{Acknowledgements}

We are grateful for the technical support of Deborah Hawkins. The preparation of this manuscript was supported by funds from the Center for Research on Genomics and Global Health, NHGRI/NIH; at Case Western Reserve University by the Center for Genetic Research Ethics and Law (3P50-HG003390), and the following NIH grants 1RC1HG005789-NHGRI; UL1 RR024989 -NCRR. Its contents are solely the responsibility of the authors and do not necessarily represent the official view of the $\mathrm{NIH}$.

Published: 24 March 2010

\section{References}

1. Department of Health and Human Services (USA), National Institutes of Health, Office for Protection from Research Risks: Title 45, Code of Federal Regulations, Part 46 - Protection of Human Subjects (45CFR 46). Federal Register 56, Bethesda, Maryland: Department of Health and Human Services; 2001.

2. Canadian Institutes of Health Research, Natural Sciences and Engineering Research Council of Canada, Social Sciences and Humanities Research Council of Canada: Tri-Council Policy Statement: Ethical Conduct for Research Involving Humans. Ottawa, Canada: Public Works and Government Services; 1998.

3. ClOMS/WHO (Council for International Organizations of Medical Sciences/ World Health Organization): International Ethical Guidelines for Biomedical Research Involving Human Subjects. Geneva, Switzerland: World Health Organization/CIOMS; 2002.

4. Nuffield Council of Bioethics: The Ethics of Healthcare in Developing Countries. London: Nuffield Council of Bioethics; 2002

5. World Medical Association: Declaration of Helsinki, Adopted by the WMA General Assembly, Helsinki, 1964, Amended 1975, 1983, 1989, 1996, 2000, 2002, 2004, 2008. [http://www.wma.net/en/30publications/10policies/b3/ index.html]

6. World Health Organization (WHO): Genomics and World Health: Report of the Advisory Committee on Health Research. Geneva, Switzerland: World Health Organization; 2002. [www.who.int/gb/ebwha/pdf_files/WHA57/A57_16-en. pdf]

7. US Genetic Nondiscrimination Information Act (GINA) of 2008, P.L 110-233, 122 Stat. 881, Order Code RL34584 (2008).

8. Coriell Cell Repositories, Coriell Institute for Medical Research (CIMR): Policy for the Responsible Collection, Storage, and Research Use of Samples from Named Populations for the NIGMS Human Genetic Cell Repository. Camden, New Jersey: CIMR; 2004.

9. Reaffirmation and Extension of NHGRI Rapid Data Release Policies: Large-scale Sequencing and other Community Resource Projects [http://www.genome.gov/10506537]

10. Bathe OF, McGuire AL: The ethical use of existing samples for genome research. Genet Med 2009, 11:712-715.

11. GAIN Collaborative Research Group, Manolio TA, Rodriguez LL, Brooks L, Abecasis G; Collaborative Association Study of Psoriasis, Ballinger D, Daly M, Donnelly P, Faraone SV; International Multi-Center ADHD Genetics Project, Frazer K, Gabriel S, Gejman P: Molecular Genetics of Schizophrenia Collaboration, Guttmacher A, Harris EL, Insel T, Kelsoe JR; Bipolar Genome Study, Lander E, McCowin N, Mailman MD, Nabel E, Ostell J, Pugh E, Sherry S, Sullivan PF; Major Depression Stage 1 Genomewide Association in Population-Based Samples Study, Thompson JF, et al:: New models of collaboration in genome-wide association studies: the Genetic Association Information Network. Nat Genet 2007, 39:1045-1051.

12. World Health Organization: Genetic Databases: Assessing the Benefits and Impact on Human and Patient Rights. Geneva, Switzerland: World Health Organization; 2003

13. Icelandic National Bioethics Committee (INBC): Guidelines Concerning Informed Consent for Participation in Genetic Research Projects or other Projects Utilizing Biological Samples. Reykjavik, Iceland: INBC; 2005.

14. Botkin J: Protecting the privacy of family members in survey and pedigree research. JAMA 2001 285:207-211. 
15. Beskow LM, Burke W, Merz JF, Barr PA, Terry S, Penchaszadeh VB, Gostin LO, Gwinn M, Khoury MJ: Informed consent for population-based research involving genetics. JAMA 2001, 286:2315-2321

16. Mascalzoni D, Hicks A, Pramstaller P, Wjst M: Informed consent in the genomics era. PLoS Med 2008, 5:e192.

17. McGuire AL, Caulfield T, Cho MK: Research ethics and the challenge of whole-genome sequencing. Nat Rev Genet 2008, 9:152-156.

18. Caulfield T, McGuire AL, Cho M, Buchanan JA, Burgess MM, Danilczyk U, Diaz CM, Fryer-Edwards K, Green SK, Hodosh MA, Juengst ET, Kaye J, Kedes L, Knoppers BM, Lemmens T, Meslin EM, Murphy J, Nussbaum RL, Otlowski M, Pullman D, Ray PN, Sugarman J, Timmons M: Research ethics recommendations for whole-genome research: consensus statement. PLoS Biol 2008, 6:e73.

19. International HapMap Project [http://hapmap.ncbi.n/m.nih.gov/thehapmap. html.en]

20. ClinSeq: A Large-Scale Medical Sequencing Clinical Research Pilot Study [http://www.genome.gov/25521306]

21. Biesecker LG, Mullikin JC, Facio FM,Turner C, Cherukuri PF, Blakesley RW, Bouffard GG, Chines PS, Cruz P, Hansen NF, Teer JK, Maskeri B, Young AC, NISC Comparative Sequencing Program, Manolio TA, Wilson AF, Finkel T, Hwang P, Arai A, Remaley AT, Sachdeev V, Shamburek R, Cannon RO, Green ED: The ClinSeq Project: piloting larage-scale genome sequencing for research in genomic medicine. Genome Res 2009, 19:1665-1674.

22. dbGaP [http://www.ncbi.nlm.nih.gov/gap]

23. Policy for Sharing of Data Obtained in NIH Supported or Conducted Genome-Wide Association Studies (GWAS) [http://grants.nih.gov/grants/ guide/notice-files/NOT-OD-07-088.html]

24. Parker M, Bull S, deVries J, Agbenyega T, Doumbo O, Kwiatkowski DP: Ethical data release in genome-wide association studies in developing countries. PLoS Med 2009, 6:e1000143

25. Coriell Institute for Medical Research [http://www.coriell.org]

26. Framingham Heart Study [http://www.framinghamheartstudy.org]

27. The Jackson Heart Study [http://jhs.jsums.edu/jhsinfo]

28. Murphy J, Scott J, Kaufman D, Geller G, LeRoy L, Hudson K: Public expectations for return of results from large-cohort genetic research. Am J Bioeth 2008, 8:36-43.

29. Kruer M: The incidentalome. JAMA 2006, 296:2801; author reply 2801-2802.

30. Parker LS: The future of incidental findings: should they be viewed as benefits? J Law Med Ethics 2008, 36:341-351, 213.

31. Clayton EW: Incidental findings in genetics research using archived DNA. J Law Med Ethics 2008, 36:286-291, 212.

32. Cho MK: Understanding incidental findings in the context of genetics and genomics. J Law Med Ethics 2008, 36:280-285, 212.

33. Marshall PA: The individual and the community in international genetic research. J Clin Ethics 2004, 15:76-86.

34. Marshall PA: Informed consent in international health research. J Empir Res Hum Res Ethics 2006, 1:25-42

35. Molyneux CS, Peshu N, Marsh K: Understanding of informed consent in a low-income setting: three case studies from the Kenyan Coast. Soc Sci Med 2004, 59:2547-2559.

36. Molyneux CS, Wassenaar DR, Peshu N, Marsh K: "Even if they ask you to stand by a tree all day, you will have to do it (laughter)...!': Community voices on the notion and practice of informed consent for biomedical research in developing countries. Soc Sci Med 2005, 61:443-454.

37. Bhutta ZA: Beyond informed consent. Bull World Health Organ 2004, 82:771-777

38. Tekola F, Bull SJ, Farsides B, Newport MJ, Adeyemo A, Rotimi CN, Davey G: Tailoring consent to context: designing an appropriate consent process for a biomedical study in a low income setting. PLOS Negl Trop Dis 2009, 3:e482.
39. Marshall PA, Adebamowo CA, Adeyemo AA, Ogundiran TO, Vekich M, Strenski T, Zhou J, Prewitt TE, Cooper RS, Rotimi CN: Voluntary participation and informed consent to international genetic research. Am J Public Health 2006, 96:1989-1995.

40. Dawson L, Kass NE: Views of US researchers about informed consent in international collaborative research. Soc Sci Med 2005, 61:1211-1222.

41. Sugarman J, Popkin B, Fortney J, Rivera R: International perspectives on protecting human research subjects. In Ethical and Policy Issues in International Research: Clinical Trials in Developing Countries Vol I, II (E-1-30). Bethesda, Maryland: National Bioethics Advisory Committee; 2001.

42. Tekola F, Bull S, Farsides B, Newport MJ, Adeyemo A, Rotimi CN, Davey G: Impact of social stigma on the process of obtaining informed consent for genetic research on podoconiosis: a qualitative study. BMC Med Ethics 2009, 10:13.

43. Tindana $\mathrm{PO}$, Kass $\mathrm{N}$, Akweongo $\mathrm{P}$ : The informed consent process in a rural African Setting: a case study of the Kassena-Nankana district of Northern Ghana. IRB 2006, 28:1-6.

44. Burgess M: Proposing modesty for informed consent. Soc Sci Med 2007, 65:2284-2295

45. Emanuel E, Wendler D, Killen J, Grady C: What makes clinical research in developing countries ethical? The benchmarks of ethical research. J Infect Dis 2004, 189:932-937.

46. Chokshi DA, Kwiatkowski DP: Ethical challenges of genomic epidemiology in developing countries. Genomics Soc Policy 2005, 1:1-15.

47. Lavery J, Grady C, Wahl E, Emanuel E: Ethical Issues in International Biomedical Research. Case 2: Selling Genes. Oxford: Oxford University Press; 2007:43-63.

48. Till J, Tritchler D: What might Tonga learn from Iceland? In Ethical Issues in International Biomedical Research. Edited by Lavery J, Grady C, Wahl E, Emanuel E. Oxford: Oxford University Press; 2007:46-52.

49. Senituli L, Boyes M: Whose DNA? Tonga and Iceland, biotech, ownership and consent. In: Ethical Issues in International Biomedical Research. Edited by Lavery J, Grady C, Wahl E, Emanuel E. Oxford: Oxford University Press; 2007:52-63.

50. Sharp RR, Foster MW: Involving study populations in the review of genetic research. J Law Med Ethics 2000, 28:41-51, 3 .

51. National Aboriginal Health Organization First Nations Centre: First Nations Regional Longitudinal Health Survey: Cost of Research Ethics. Revised edition. Ottawa: NAHO; 2005

52. Indigenous Peoples' Health Research Centre (IPHRC): The Ethics of Research Involving Indigenous Peoples. Saskatchewan: IPHRC; 2004.

53. American Indian Law Center: Model Tribal Research Code, with Materials for Tribal Research and Checklist for Indian Health Boards. 3rd edition. Albuquerque: American Indian Law Center; 1999.

54. Australian Institute of Aboriginal and Torres Strait Islander Studies, Dept. of Education, Science and Training: Guidelines for Ethical Research in Indigenous Studies. Canberra: AIATSIS; 2000.

55. Maori Health Committee of the Health Research Council (HRC) of New Zealand: Guidelines for Researchers on Health Research Involving Maori. Wellington, NZ: HRC; 1998.

56. Rotimi C, Dunston G, Berg K, Akinsete O, Amoah A, Owusu S, Acheampong J, Boateng K, Oli J, Okafor G, Osotimehin B, Abbiyesuku F, Johnson T, FurbertHarris P, Kittles R, Vekich M, Adegoke O, Bonney G, Collins F: In search of susceptibility genes for type 2 diabetes in West Africa: the design and results of the first phase of the AADM Study. Ann Epidemiol 2001, 11:51-58.

doi:10.1186/gm14

Cite this article as: Rotimi CN, Marshall PA: Tailoring the process of informed consent in genetic and genomic research. Genome Medicine 2010, 2:20. 